\title{
Physical activity and dietary behaviors of health clinic workers trying to lose weight
}

\author{
Tan Leng Goh*, Trever Ball, Janet M. Shaw, James C. Hannon
}

Department of Exercise and Sport Science, University of Utah, Salt Lake City, USA; ${ }^{*}$ Corresponding Author: tanleng.goh@utah.edu

Received 29 May 2012; revised 27 June 2012; accepted 8 July 2012

\begin{abstract}
Health clinic workers are potential agents of change for weight loss to patients, yet their behaviors are not well known. This study examined physical activity (PA) levels and dietary habits of health clinic workers who were and who were not trying to lose weight. Participants were 64 community health clinic workers (58 females and 6 males). Moderate-to-vigorous intensity (MVI) time spent in PA was assessed by triaxial accelerometry over 7 consecutive days. Dietary habits and weight loss efforts were determined by a food frequency questionnaire. Differences in MVI and nutrition variables were assessed by one-way ANOVA, comparing those trying to lose weight and those not trying to lose weight. 48 out of 64 health clinic workers (approximately $75 \%$ ) indicated that they were currently trying to lose weight. There were significant differences $(p<0.05)$ in Body Mass Index (BMI), daily energy (Kcal) and fat $(\mathrm{g})$ intake between those trying to lose weight and those not trying to lose weight. There were no significant differences in MVI, daily sugar intake (g), vegetable and fruit servings, and daily fiber intake (g) between groups. Health clinic workers trying to lose weight appear to be engaging in some appropriate dietary but not PA behaviors.
\end{abstract}

Keywords: Accelerometer; Overweight; Healthcare; Nutrition

\section{INTRODUCTION}

Adult and childhood obesity has become an epidemic in the United States and in many other developed countries. Currently, one-third of US adults are obese, with 72 percent of men and 64 percent of women being overweight or obese due to poor diet and physical inactivity [1]. Inactivity or low levels of physical activity (PA) among adolescents and adults are common in the US population $[2,3]$. These behaviors are major contributors to morbidity and mortality from cardiovascular disease, hypertension, type 2 diabetes, osteoporosis, and some types of cancer $[1,3]$. Conversely, adults who engage in regular PA, eat a healthy diet and avoid becoming overweight, will have a markedly lower morbidity and mortality when compared to their less active counterparts $[4,5]$.

Weight reduction is encouraged by major health agencies because it reduces health risks associated with chronic disease $[1,6]$. According to the Dietary Guidelines for Americans 2010, adults who are trying to lose weight are recommended to maintain a dietary pattern low in caloric density, characterized by a high intake of vegetables, fruit, and dietary fiber and a low intake of total fat, saturated fat, and added sugars [1]. Successful weight loss and weight maintenance strategies reported by more than 4800 participants from the National Weight Control Registry include eating a relatively low-fat diet, eating breakfast almost every day, weighing regularly, and engaging in high levels of PA for about 1 hour per day [7]. In fact, moderate-intensity PA between 150 and 250 minutes per week only provide modest weight loss; greater amounts of PA of more than 250 minutes per week are associated with clinically significant weight loss [6]. The benefits of high intensity physical activities in maintaining weight loss is greater among overweight or obese than normal weight individuals [8]. Maintaining high levels of activity are also associated with smaller age-related gains in Body Mass Index (BMI) and waist circumference [9].

The increasing prevalence of obesity makes it important that primary care providers and health clinic workers take action in counseling overweight patients [10]. Professional organizations recommend that brief counseling be delivered to promote PA and healthy eating in routine clinical encounters $[11,12]$. Other sources of PA counseling to patients come from allied health professionals and nurses $[13,14]$. However, most nurses only counsel patients on PA approximately once a year through casual discussion rather than systematic procedures [14]. Even though PA counseling during patient visits was reported 
mostly in office-based practices than in hospital outpatient clinics, the rates of such counseling are relatively low in both settings [15]. Because of the lack of time in clinic settings for counseling, the promotion of PA and healthy diet behaviors through the modeling of health clinic workers in relation to weight control may be a source of influence to the patients.

Even though health clinic workers have the potential to influence their patients in a clinic setting, little is known about their PA and diet patterns in relation to their weight control [16]. Additionally, many studies on health clinic workers rely on self-reported measures of PA patterns [17-19]. Although self-reported PA questionnaires may provide the approximate level of PA at a population level, they may not determine whether an individual is participating in the intensity and duration of PA advocated in current public health recommendations [20]. More objective measures are necessary to study the PA patterns of health clinic workers. Therefore, the primary aim of this study was to examine the PA levels and dietary habits of health clinic workers who were and who were not trying to lose weight. A secondary aim was to compare our participants' dietary patterns with the recommended Dietary Guidelines for Americans 2010 [1].

\section{METHODS}

\subsection{Participants and Settings}

This study took place within the context of a larger study designed to validate new PA and diet instruments developed for use in a primary care setting. A total of 88 health clinic workers were recruited for the study. Those with serious health conditions that would preclude normal PA were excluded. Two participants were ineligible due to current pregnancy and 13 participants discontinued participation due to lack of interest or time. Seventy five participants provided complete dietary data resulting in a retention rate of $85.2 \%$. Of the 75 participants, 64 (58 women and 6 men; $\mathrm{M} \pm \mathrm{SD}$ age of $37.3 \pm 11.4$ years) were in compliance with the accelerometer protocol guidelines. Forty-seven percent of the participants self reported as Latino/a, while $53 \%$ self reported as Non Latino/a. All participants were staff employees at one of three Community Health Centers (CHC Inc.) or one of four University of Utah Community Clinics, all of which focused on primary care and were located in a large Southwestern City and surrounding area. The Institutional Review Board approved all study procedures and all participants provided written, informed consent.

\subsection{Instrumentation and Measures}

Participants provided medical history and demographic data including age, sex, height, weight, whether they were responsible for providing or assisting with patient care, hours they work each week, and educational level. Participants' BMI was calculated from self-reported height and weight.

\subsection{Physical Activity}

The PA patterns of our participants were measured using the ActiGraph GT3X accelerometers set for 60 -s recording epoch periods, which is the most frequent setting for adults [21]. The triaxial accelerometer is a valid and reliable measurement of MVI time spent in PA in freeliving conditions [22]. Participants were instructed to wear the accelerometers for 7 consecutive days during waking hours. They were advised to wear it on the same place on their waist each day and not to wear it when they were showering or doing water exercises. MVI time spent in PA was calculated using the cutpoint of $>1951$ ct. $\min ^{-1}$ [23]. 3 - 5 days of accelerometer monitoring is required to reliably estimate habitual PA among adults [24]. Adults are recommended to perform bouts of MVI time in PA lasting at least 10 minutes for health benefits [25]. Therefore, participants with at least 4 days of accelerometer data and at least 10 minutes accrued bouts of MVI time in PA were used for data analysis. Non-bout PA was also analyzed to compare our findings with other studies. The Compendium of Physical Activities was used to impute MVI time in PA of water-based exercises reported by the participants in the accelerometer log [26].

\subsection{Diet Patterns}

The diet patterns of our participants were measured using the Block Food Frequency Questionnaire (BFFQ) [27]. The BFFQ is a valid instrument to measure diet behaviors of participants [27]. Because we had a large percentage of participants who self-reported as Latino/a, we used both the English version for a typical American diet and the bilingual Spanish-English version for a typical Hispanic diet, which provide identical analyses based upon culturally derived food lists [28]. The participants chose either the English or the Spanish version that would best reflect their eating patterns. Participants were asked to respond to the BFFQ in a manner that would capture their habitual intake for the past year. Participants also self-reported their ethnicity as either Latino/a or Non Latino/a, their health status, as well as whether they were currently trying to lose weight on the BFFQ. For the purposes of this study, daily intake of energy (Kcal), total sugar ( $\mathrm{g}$ ), percent of calories from sweets, total fat (g), percent of calories from fat, number of vegetable and fruit servings, and dietary fiber $(g)$ were analyzed to examine our participants' food patterns with the Dietary Guidelines for Americans 2010 that were relevant to weight loss. 


\subsection{Procedures}

Data collection spanned from January to September 2010. During the first visit with the participants at their clinics, we explained the study protocol and obtained informed consent. Then, we gave the BFFQs, accelerometers and an accelerometer log to consented participants. They recorded the time they put on the accelerometer in the morning and when they removed it in the evening. They also recorded any water exercise or instances such as sickness that prevented them from wearing the accelerometer or from doing usual PA. After 1 week, we collected the BFFQs, accelerometers and the accelerometer log from the participants. The BFFQs were checked for missing responses. Data from the returned accelerometers were downloaded immediately after the second visit with ActiLife software version 5.7.0 (ActiGraph, Pensacola, FL). After data collection was completed, the BFFQs were mailed to NutritionQuest in October 2010 for processing and nutrient calculation. The processed BFFQs with nutrient results were sent back to us in an electronic file in November 2010 for data analysis.

\subsection{Statistical Analysis}

A one-way ANOVA was used to compare differences in BMI, MVI time spent in PA and nutrition variables of those trying to lose weight and those not trying to lose weight. Data were entered and results generated using SPSS (Version 18.0, Chicago, IL). Descriptive data were calculated as frequencies and reported as percentages or unit values. An alpha level of 0.05 was used for all statistical tests.

\section{RESULTS}

The participants' background and demographic information are presented in Table 1. A majority of our participants were female and this corresponds with the gender breakdown of health clinic workers in most clinic settings. There was an equal split between Latino and Non Latino participants. The majority of our participants (82.8\%) were responsible for providing or assisting with patient care and self-identified as medical assistants. Over $75 \%$ of our participants reported their health status as good, very good or excellent. A majority of our participants $(87.5 \%)$ has completed some college or has a bachelor's degree. From the BFFQ, we determined that of the 64 participants, 48 of them (approximately 75\%) were trying to lose weight. Participants who were trying to lose weight worked a similar number of hours each week as those who were not trying to lose weight.

Participants who reported trying to lose weight had higher BMI $(p=0.033)$, lower average daily energy in
Table 1. Descriptive characteristics of participants.

\begin{tabular}{lcc}
\hline \multicolumn{1}{c}{ Variable } & $\begin{array}{c}\text { TLW }(n=48) \\
\mathrm{M} \pm \mathrm{SD}\end{array}$ & $\begin{array}{c}\text { NTLW }(n=16) \\
\mathrm{M} \pm \mathrm{SD}\end{array}$ \\
\hline Age & $37.5 \pm 11.3$ & $36.4 \pm 11.9$ \\
Sex & & \\
Female & $45(94 \%)$ & $13(81 \%)$ \\
Male & $3(6 \%)$ & $3(19 \%)$ \\
Ethnicity & & \\
Latino/a & 22 & 8 \\
Non Latino/a & 26 & 8 \\
Patient Care & & \\
Responsible & 41 & 12 \\
Non Responsible & 7 & 4 \\
Hours Worked Each Week & $41.6 \pm 5.8$ & $40.9 \pm 5.8$ \\
Self-Reported Health Status & & \\
Excellent & 4 & 2 \\
Very Good & 5 & 5 \\
Good & 27 & 6 \\
Fair & 11 & 2 \\
Poor & 1 & 1 \\
Education & & 1 \\
Less than High School & 1 & 1 \\
Completed High School & 5 & \\
Some College & & \\
Completed 4 Years & 35 & \\
College Degree & 7 & \\
P & & \\
\hline
\end{tabular}

$\mathrm{M}=$ Mean; $\mathrm{SD}=$ Standard deviation; TLW = Trying to lose weight; NTLW $=$ Not trying to lose weight.

take in Kcal $(p=0.025)$, and lower daily total fat intake in grams $(p=0.020)$ when compared to participants who were not trying to lose weight. The average BMI of participants who were trying to lose weight would be classified as obese while the average BMI of participants who were not trying to lose weight would be classified as overweight [29]. There were no significant differences in average daily total sugar (g) intake, percent of average calories from sweets, average percent of calories from fat, number of vegetable and fruit servings, daily total fiber intake $(\mathrm{g})$, and average weekly MVI time spent in PA between the two groups. The means and standard deviations of the measured variables are presented in Table 2.

We descriptively compared our participants' reported food intake with the recommended food patterns from the Dietary Guidelines for Americans 2010 [1], presented in Table 3. The recommended average daily energy intake ranges from 1800 to 3000 calories for adults between the ages of 20 - 50 [1]. We found that both groups of participants' average daily energy intake meet the recommended food pattern. Those trying to lose weight reported consuming fewer daily calories than those not trying to lose weight. Participants who were trying to lose weight also just meet the recommended food pattern for daily total fat $(\mathrm{g})$ and percent calories from fat, whereas those who were not trying to lose weight consumed more 
Table 2. Dietary and PA differences between participants who were trying to lose weight and those not trying to lose weight.

\begin{tabular}{cccc}
\hline Variable & $\begin{array}{c}\text { TLW }(n=48) \\
\mathrm{M} \pm \mathrm{SD}\end{array}$ & $\begin{array}{c}\mathrm{NTLW}(n=16) \\
\mathrm{M} \pm \mathrm{SD}\end{array}$ & $\mathrm{p}$ value \\
\hline BMI & $31.6 \pm 6.6$ & $27.2 \pm 8.5$ & $0.033^{*}$ \\
Daily Energy (Kcal) & $1566 \pm 679$ & $2052 \pm 877$ & $0.025^{*}$ \\
Daily Total Sugar (g) & $95.8 \pm 54.9$ & $104.8 \pm 72.1$ & 0.605 \\
\% of Cal from Sweets & $15.1 \pm 9.3$ & $14.1 \pm 8.4$ & 0.688 \\
Daily Total Fat (g) & $60.8 \pm 28.6$ & $81.0 \pm 30.9$ & $0.020^{*}$ \\
\% of Cal from Fat & $34.9 \pm 5.1$ & $36.1 \pm 6.3$ & 0.434 \\
Vegetable Servings & $2.0 \pm 1.8$ & $2.3 \pm 1.3$ & 0.526 \\
Fruit Servings & $1.1 \pm 0.8$ & $1.1 \pm 0.6$ & 0.891 \\
$\begin{array}{c}\text { Daily Total Fiber (g) } \\
\text { Weekly Bout MVI (min) }\end{array}$ & $15.0 \pm 7.6$ & $18.7 \pm 7.8$ & 0.101 \\
$\begin{array}{c}\text { Weekly Non-Bout MVI } \\
\text { (min) }\end{array}$ & $121.3 \pm 101.1$ & $133.0 \pm 95.1$ & 0.684 \\
\hline
\end{tabular}

$\mathrm{M}=$ Mean; $\mathrm{SD}=$ Standard deviation; $\mathrm{TLW}=$ Trying to lose weight; NTLW $=$ Not trying to lose weight; $\mathrm{BMI}=$ Body mass index $; \mathrm{Cal}=$ Calories; $\mathrm{MVI}$

$=$ Moderate-to-vigorous intensity; Significantly different at ${ }^{*} \mathrm{p}<0.05$.

Table 3. Comparison of our findings with the recommended food intake.

\begin{tabular}{cccc}
\hline Food Groups & $\begin{array}{c}\text { USDA Food } \\
\text { Pattern }\end{array}$ & $\begin{array}{c}\text { TLW }(n=48) \\
\mathrm{M} \pm \mathrm{SD}\end{array}$ & $\begin{array}{c}\text { NTLW }(n=16) \\
\mathrm{M} \pm \mathrm{SD}\end{array}$ \\
\hline Daily Total Fat (g) & 65 & $60.8 \pm 28.6$ & $81.0 \pm 30.9$ \\
\% of Cal from Fat & $20-35$ & $34.9 \pm 5.1$ & $36.1 \pm 6.3$ \\
Vegetable Servings & 2.5 & $2.0 \pm 1.8$ & $2.3 \pm 1.3$ \\
Fruit Servings & 2 & $1.1 \pm 0.8$ & $1.1 \pm 0.6$ \\
Daily Total Fiber (g) & $25-34$ & $15.0 \pm 7.6$ & $18.7 \pm 7.8$ \\
\hline
\end{tabular}

$\mathrm{M}=$ Mean; SD = Standard deviation; TLW = Trying to lose weight; NTLW $=$ Not trying to lose weight; $\mathrm{Cal}=$ Calories; ${ }^{*}=$ Based on a 2000 calorie diet.

than recommended. Both groups of participants consumed less than the recommended number of daily vegetable and fruit servings, and daily total fiber $(\mathrm{g})$ intake.

\section{DISCUSSION}

The primary purpose of this study was to examine the PA levels and dietary habits of health clinic workers who were and who were not trying to lose weight. We hypothesized that health clinic workers who were trying to lose weight would maintain a higher level of PA and healthier dietary habits than workers who were not trying to lose weight. Overall, we found that a majority of our participants $(75 \%)$ were trying to lose weight and the BMI of those who were trying to lose weight were significantly higher than those who were not trying to lose weight. The dietary behaviors of our participants who were trying to lose weight are slightly different from those who were not trying to lose weight, but their PA behaviors are similar. Particularly, those who were trying to lose weight reported consuming fewer daily calories (Kcal) and daily total fat (g) than those who were not trying to lose weight. However, there were no significant differences in the weekly MVI time spent in PA between the two groups. Our secondary purpose was to compare our participants' dietary habits with the Dietary Guidelines for Americans 2010 [1]. We found that our participants who were trying to lose weight meet the daily total fat $(\mathrm{g})$ and percent calories from fat recommendations, whereas those who were not trying to lose weight consumed more than recommended. However, both groups of participants consumed lesser fruits and vegetables servings, and lesser daily total fiber than the food pattern recommendations.

Many studies suggest that engaging in high levels of PA for at least 250 minutes per week will help in losing and controlling weight [6-9]. However, we found that our participants who were trying to lose weight did not engage in sufficient PA that will aid them in losing or controlling their weight. In fact, the weekly bout MVI time in PA by participants who were trying to lose weight $(37.1 \pm 76.3)$ and participants who were not trying to lose weight $(39.5 \pm 63.5)$ were much lower than the recommended 150 minutes of weekly bout MVI time in PA for health maintenance [25]. In addition, the weekly nonbout MVI time in PA by participants who were trying to lose weight $(121.3 \pm 101.1)$ and participants who were not trying to lose weight $(133.0 \pm 95.1)$ were lower than the recommendations by the American College of Sports Medicine and the American Heart Association, and other studies that support engaging in at least 250 minutes of PA per week for successful weight loss $[6,7,25]$.

Our findings are in contrast to the study of PA behaviors among National Weight Control Registry (NWCR) participants, who have demonstrated successful weight loss. The NWCR participants who were trying to lose weight spent more time per day in sustained bouts of MVI than overweight and normal weight participants in control groups [30]. Nonetheless, our findings are consistent with other studies in that our participants who were trying to lose weight seem to engage in dietary strategies, instead of increasing PA to aid in weight loss [17-19,31]. For example, participants in the National Physical Activity and Weight Loss Survey reported using more diet, but not PA or both strategies to lose weight [17]. In one study, $34.0 \%$ of men and $46.6 \%$ of women who participated in a nationwide telephone survey reported trying to lose weight, but less than half of them engaged in high volumes of PA [18]. Of those who were trying to lose weight, only $47.6 \%$ of men and $39.9 \%$ engaged in moderate or vigorous lifestyle PA at least 60 
minutes per day on 5 or more days per week [18]. Furthermore, the prevalence of engaging in high volumes of lifestyle PA was greater among Whites than among non-Whites [18]. In another study, more than half of the registered nurses from five multi-state settings who participated in an electronic survey reported being overweight, but only $50 \%$ met PA standards [19]. Kruger et al. [32] also identified that despite the emphasis of PA in their study's weight loss program, only half of the participants trying to lose weight reported using exercise, and among those, only slightly more than half met the minimal recommendations for PA.

Although we found that our participants who were trying to lose weight engaged in slightly healthier diet behavior, such as reporting lower daily total energy intake (Kcal) and daily total fat intake (g) than those who were not trying to lose weight, both groups did not meet the recommended guidelines for daily total fiber intake (g), and number of daily vegetable and fruit servings. Specifically, they consumed less than the recommended proportions for vegetables, fruits and dietary fiber. Dietary habits such as consuming five or more fruits and vegetables a day are effective strategies to reduce and control weight $[7,33]$. A high intake of dietary fiber is also recommended for adults who are trying to lose weight and maintain weight [1]. Participants who were trying to lose weight only just meet the recommended guidelines for percent calories from fat, whereas those not trying to lose weight reported consuming slightly more than the recommendations. Higher percent of calories in the daily diet from animal, saturated and trans fat has strong associations with weight gain among overweight women [34]. Reduction in the amount of sugar consumption through beverages is also recommended because high consumption of sugar-sweetened beverages is associated with a greater magnitude of weight gain and an increased risk for development of type 2 diabetes [35]. Such beverages provide excessive calories and large amounts of rapidly absorbable sugars [35]. Regular consumption of sugar-sweetened beverages is associated with a higher risk of coronary heart diseases in women [36]. Though we do not have a direct comparison of the daily total sugar $(\mathrm{g})$ with the recommended guidelines, we found that our participants' consumption of daily total sugar $(\mathrm{g})$ is much higher than the recommended $32(\mathrm{~g})$ of added sugars per day [1].

Obesity has become an epidemic in the United States and therefore weight reduction is encouraged because it reduces health risks associated with chronic disease [6]. Health care providers are potential advocates of PA and healthy dietary habits to their patients $[11,12,16,37]$. Physicians who are regularly physically active have a stronger influence on their patients' PA behavior and are more likely to recommend PA to their patients than phy- sicians who are inactive [38]. Therefore, physicians who engage in these behaviors are viewed as more credible sources of PA recommendations and hence are potential PA role models to their patients [38]. Because health clinic workers may be similar to patients in terms of language and culture, they may also have an influence on the patients in terms of PA and healthy diet role modeling. Additionally, the majority of our participants self-identified as medical assistants and they are ideally suited to provide linguistically and culturally concordant coaching that can address lifestyle changes [39]. Therefore, more efforts are needed to help those trying to lose weight incorporate appropriate levels of PA and healthy diet patterns into their weight loss strategy [32]. Likewise, through education, health clinic workers can also be better informed of the benefits of PA and behavioral strategies to incorporate PA into their daily living. This is important in a clinic setting, because health clinic workers can further advocate engaging in PA as a means of reducing and controlling weight to their patients.

Although this study addresses a gap in the literature regarding health clinic workers dietary and PA behaviors in relation to weight loss, it is not without limitations. One limitation of the study is that the BFFQ ultimately relies on self-report, whereas the accelerometry does not. Even though the accelerometry is an objective measure of the participants' PA behaviors, we relied on them to wear the accelerometers in compliance with our instructions. A second limitation is that the BFFQ reflects habitual dietary intake over the past year, whereas the accelerometry data revealed their PA habits over the past week, and we assume that these PA habits are habitual.

In view of the potential influence and impact health clinic workers have on their patients in healthy dietary and PA habits, we suggest several considerations for future research. Future research may determine whether the attitudes and strategies engaged toward weight loss of health clinic workers who are involved in patient care will impact patients. Specifically, future researchers can investigate whether workers involved in patient care will make weight loss recommendations to patients similar to what they do themselves and whether this phenomenon also holds true for workers who are not involved in patient care. In addition, future researchers can investigate whether health clinic workers follow appropriate behaviors for weight loss so as to set an example to help others who are also trying to lose weight. Role modeling by health clinic workers will become increasingly important as recent Medicare legislation for obesity counseling becomes a regular practice in primary care [40]. Another future research direction will be to examine barriers and hence, devise strategies to assist health clinic workers in achieving the recommended levels of PA and diet patterns to lose weight. A health intervention program may 
be effective to assist health clinic workers to make healthier and better choices towards their lifestyle [41]. Finally, a small percentage (25\%) of our participants reported that they were not trying to lose weight, even though they are considered as overweight based on their BMI. Future researchers can investigate whether overweight participants are aware of their weight status and examine the reasons why they are not trying to lose weight.

Given that there is a paucity of studies addressing health clinic workers' PA and diet behaviors in relation to their weight loss, there is a need for more studies in this area. Consequently, this will help promote appropriate PA and diet behaviors in health care settings, which has the potential to have tremendous impact on a large number of patients.

\section{ACKNOWLEDGEMENTS}

This research was funded in part by DHHS Office on Women's Health (ASTWH070006-01-00) and the University of Utah Undergraduate Research Opportunities Program (UROP). We would like to thank the University of Utah Primary Care Research Network, the participating University of Utah Community Clinics, and Community Health Centers, Inc., Nicole Major, Abe Schlauderaff and Danita Arguello for their assistance.

\section{REFERENCES}

[1] US Department of Agriculture, US Department of Health and Human Services (2010) Dietary guidelines for Americans. 7th Edition, US Government Printing Office, Washington DC.

[2] Bassett, D.R., Pucher, J., Buehler, R., Thompson, D.L. and Crouter S.E. (2008) Walking, cycling, and obesity rates in Europe, North America, and Australia. Journal of Physical Activity and Health, 5, 795-814.

[3] Carnethon, M.R., Gulati, M. and Greenland, P. (2005) Prevalence and cardiovascular disease correlates of low cardiorespiratory fitness in adolescents and adults. The Journal of American Medical Association, 294, 29812988. doi:10.1001/jama.294.23.2981

[4] van Dam, R.M., Li, T., Spiegelman, D., Franco, O.H. and Hu, F.B. (2008) Combined impact of lifestyle factors on mortality: Prospective cohort study in US women. British Medical Journal, 337, a1440. doi:10.1136/bmj.a1440

[5] Chiuve, S.E., Fung, T.T., Rexrode, K.M., et al. (2011) Adherence to a low-risk, healthy lifestyle and risk of sudden cardiac death among women. The Journal of American Medical Association, 306, 62-69. doi:10.1001/jama.2011.907

[6] Donnelly, J.E., Blair, S.N., Jakicic, J.M., et al. (2009) American college of sports medicine position stand. Appropriate physical activity intervention strategies for weight loss and prevention of weight regain for adults. Medicine and Science in Sport and Exercise, 41, 459-471. doi:10.1249/MSS.0b013e3181949333
[7] Hill, J.O., Wyatt, H., Phelan, S. and Wing, R. (2005) The national weight control registry: Is it useful in helping deal with our obesity epidemic? Journal of Nutrition Education and Behavior, 37, 206-210. doi:10.1016/S1499-4046(06)60248-0

[8] Mekary, R.A., Feskanich, D., Hu, F.B., Willett, W.C. and Field, A.E. (2010) Physical activity in relation to longterm weight maintenance after intentional weight loss in premenopausal women. Obesity, 18, 167-174. doi:10.1038/oby.2009.170

[9] Hankinson, A.L., Daviglus, M.L., Bouchard, C., et al. (2010) Maintaining a high physical activity level over 20 years and weight gain. The Journal of American Medical Association, 304, 2603-2610. doi:10.1001/jama.2010.1843

[10] Sonntag, U., Henkel, J., Renneberg, B., Bockelbrink, A., Braun, V. and Heintze, C. (2010) Counseling overweight patients: Analysis of preventive encounters in primary care. International Journal for Quality in Health Care, 22, 486-492. doi:10.1093/intqhe/mzq060

[11] Jacobson, D.M., Strohecker, L., Compton, M.T. and Katz, D.L. (2005) Physical activity counseling in the adult primary care setting: Position statement of the American College of Preventive Medicine. American Journal of Preventive Medicine, 29, 158-162. doi:10.1016/j.amepre.2005.04.009

[12] Fletcher, G.F. (1997) How to implement physical activity in primary and secondary prevention. A statement for healthcare-professionals from the task force on risk-reduction. American Heart Association. Circulation, 96, 355-357. doi:10.1161/01.CIR.96.1.355

[13] Tulloch, H., Fortier, M. and Hogg, W. (2006) Physical activity counseling in primary care: Who has and who should be counseling? Patient Education and Counselling, 64, 6-20. doi:10.1016/j.pec.2005.10.010

[14] Buchholz, S.W. and Purath, J. (2007) Physical activity and physical fitness counseling patterns of adult nurse practitioners. Journal of American Academy of Nurse Practitioners, 19, 86-92. doi:10.1111/j.1745-7599.2006.00197.x

[15] Lin, S.X., Hyman, D. and Larson, E. (2005) Provision of health counseling in office-based practices and hospital outpatient clinics. Preventive Medicine, 40, 542-546. doi:10.1016/j.ypmed.2004.06.015

[16] Wilcox, S., Parra-Medina, D., Felton, G.M., Poston, M.B. and McClain, A. (2010) Adoption and implementation of physical activity and dietary counseling by community health center providers and nurses. Journal of Physical Activity and Health, 7, 602-612.

[17] Millstein, R.A., Carlson, S.A., Fulton, J.E., et al. (2008) Relationships between body size satisfaction and weight control practices among US adults. The Medscape Journal of Medicine, 10, 119.

[18] Kruger, J., Yore, M.M., Ainsworth, B.E. and Macera, C.A. (2008) Physical activity patterns associated with weightcontrol status: Differences by race and sex. Journal of Physical Activity and Health, 5, 456-468.

[19] Tucker, S.J., Harris, M.R., Pipe, T.B. and Stevens, S.R. (2010) Nurses' ratings of their health and professional 
work environments. American Association of Occupational Health Nurses Journal, 58, 253-267.

[20] Loney, T., Standage, M., Thompson, D., Sebire, S.J. and Cumming, S. (2011) Self-report vs objectively assessed physical activity: Which is right for public health? Journal of Physical Activity and Health, 8, 62-70.

[21] Ainsworth, B.E., Bassett, D.R., Strath, S.J., et al. (2000) Comparison of three methods for measuring the time spent in physical activity. Medicine and Science in Sport and Exercise, 32, S457-464. doi:10.1097/00005768-200009001-00004

[22] McClain, J.J., Sisson, S.B. and Tudor-Locke, C. (2007) Actigraph accelerometer interinstrument reliability during free-living in adults. Medicine and Science in Sport and Exercise, 39, 1509-1514. doi:10.1249/mss.0b013e3180dc9954

[23] Freedson, P.S., Melanson, E. and Sirard, J. (1998) Calibration of the computer science and applications, Inc. accelerometer. Medicine and Science in Sport and Exercise, 30, 777-781.

[24] Trost, S.G., McIver, K.L. and Pate, R.R. (2005) Conducting accelerometer-based activity assessments in fieldbased research. Medicine and Science in Sport and Exercise, 37, S531-543. doi:10.1097/00005768-199805000-00021

[25] Haskell, W.L., Lee, I.M., Pate, R.R., et al. (2007) Physical activity and public health: Updated recommendation for adults from the American College of Sports Medicine and the American Heart Association. Circulation, 116, 1081-1093. doi:10.1161/CIRCULATIONAHA.107.185649

[26] Ainsworth, B.E., Haskell, W.L., Herrmann, S.D., et al. (2011) 2011 compendium of physical activities: A second update of codes and MET values. Medicine and Science in Sport and Exercise, 43, 1575-1581. doi:10.1249/MSS.0b013e31821ece12

[27] Block, G., Woods, M., Potosky, A. and Clifford, C. (1990) Validation of a self-administered diet history questionnaire using multiple diet records. Journal of Clinical Epidemiology, 43, 1327-1335. doi:10.1016/0895-4356(90)90099-B

[28] Block, G., Wakimoto, P., Jensen, C., Mandel, S. and Green, R.R. (2006) Validation of a food frequency questionnaire for Hispanics. Preventing Chronic Disease, 3, 110.

[29] Centers for disease control and prevention. About BMI for adults. http://www.cdc.gov/healthyweight/assessing/bmi/adult b $\underline{\text { mi/index.html }}$

[30] Catenacci, V.A., Grunwald, G.K., Ingebrigtsen, J.P., et al. (2011) Physical activity patterns using accelerometry in the national weight control registry. Obesity, 19, 11631170. doi:10.1038/oby.2010.264

[31] Green, K.L., Cameron, R., Polivy, J., et al. (1997) Weight dissatisfaction and weight loss attempts among Canadian adults. Canadian Medical Association Journal, 157, S17S25.

[32] Kruger, J., Galuska, D.A., Serdula, M.K. and Kohl, H.W. (2005) Physical activity profiles of US adults trying to lose weight: NHIS 1998. Medicine and Science in Sport and Exercise, 37, 364-368.

doi:10.1249/01.MSS.0000155434.87146.72

[33] Kruger, J., Blanck, H.M. and Gillespie, C. (2008) Dietary practices, dining out behavior, and physical activity correlates of weight loss maintenance. Preventing Chronic Disease, 5, A11.

[34] Field, A.E., Willett, W.C., Lissner, L. and Colditz, G.A. (2007) Dietary fat and weight gain among women in the nurses' health study. Obesity, 15, 967-976. doi:10.1038/oby.2007.616

[35] Schulze, M.B., Manson, J.E., Ludwig, D.S., et al. (2004) Sugar-sweetened beverages, weight gain, and incidence of type 2 diabetes in young and middle-aged women. The Journal of American Medical Association, 292, 927-934. doi:10.1001/jama.292.8.927

[36] Fung, T.T., Malik, V., Rexrode, K.M., Manson, J.E., Willett, W.C. and Hu, F.B. (2009) Sweetened beverage consumption and risk of coronary heart disease in women. The American Journal of Clinical Nutrition, 89, 1037-1042. doi:10.3945/ajen.2008.27140

[37] Halm, J. and Amoako, E. (2008) Physical activity recommendation for hypertension management: Does healthcare provider advice make a difference? Ethnicity and Disease, 18, 278-282.

[38] Harsha, D.M., Saywell, R.M., Thygerson, S. and Panozzo, J. (1996) Physician factors affecting patient willingness to comply with exercise recommendations. Clinical Journal of Sport Medicine, 6, 112-118. doi:10.1097/00042752-199604000-00009

[39] Bennett, H.D., Coleman, E.A., Parry, C., Bodenheimer, T. and Chen, E.H. (2010) Health coaching for patients with chronic illness. Family Practice Management, 17, 24-29.

[40] US Department of Health and Human Services. Decision memo for intensive behavioral therapy for obesity. http://www.cms.gov/medicare-coverage-database/details/ nca-decision-memo.aspx?\&NcaName=Intensive $\% 20 \mathrm{Beh}$ avioral $\% 20$ Therapy $\% 20$ for $\% 20$ Obesity $\& b c=$ ACAAAAA AIAAA\&NCAId $=253 \&$

[41] Bourne, P.A., Glen, L.V., Laws, H. and Kerr-Campbell, M.D. (2010) Health, lifestyle and health care utilization among health professionals. Health, 2, 557-565. doi:10.4236/health.2010.26083 